\title{
Erratum to: Cost Effectiveness of Paricalcitol Versus Cinacalcet with Low-Dose Vitamin D for Management of Secondary Hyperparathyroidism in Haemodialysis Patients in the USA
}

\author{
Amit Sharma - Thomas S. Marshall • \\ Samina S. Khan • Beverly Johns
}

Published online: 3 January 2014

(C) Springer International Publishing Switzerland 2013

\section{Erratum to: Clin Drug Investig \\ DOI 10.1007/s40261-013-0151-4}

A Published-Ahead-of-Print version of this article was made available online at http://link.springer.com/article/10. 1007/s40261-013-0151-4 on 09 November 2013. Errors were subsequently identified in that version of the article, and the following corrections should be noted:

Section 3.1, line 5, which previously read:

"As shown in Table 2, among US patients, 29 (56.9\%) in the paricalcitol group and $16(34.0 \%)$ in the cinacalcet group reached this endpoint (a difference of $23 \%$, $p=0.0235)$."

Should read:

"As shown in Table 3, among US patients, 29 (56.9 \%) in the paricalcitol group and $16(34.0 \%)$ in the cinacalcet group reached this endpoint (a difference of $23 \%$, $p=0.0235)$."

The online version of the original article can be found under doi:10.1007/s40261-013-0151-4.

\footnotetext{
A. Sharma

Nephrian Inc., 870 Paseo Santa Cruz, Thousand Oaks, CA 91320-6779, USA

e-mail: sharmaaxs@gmail.com

T. S. Marshall $(\bowtie) \cdot$ S. S. Khan · B. Johns

AbbVie, Inc., 1 North Waukegan Road,

North Chicago, IL 60064, USA

e-mail: t.marshall@abbvie.com

S. S. Khan

e-mail: samina.khan@abbvie.com

B. Johns

e-mail: beverly.a.johns@abbvie.com
}

Section 3.3, first paragraph, line 8, which previously read:

"Table 3 also shows the difference between the paricalcitol and cinacalcet groups in the proportions of patients who responded, based on each endpoint included (based on data in Table 2)."

Should read:

"Table 4 also shows the difference between the paricalcitol and cinacalcet groups in the proportions of patients who responded, based on each endpoint included (based on data in Table 3)."

Section 3.3, third paragraph, line 1, which previously read:

"Figure 1 is a scatterplot of all 1,000 bootstrap replicates, with the $x$-axis representing the incremental cost and the $y$-axis representing the incremental effectiveness of the paricalcitol-based regimen compared with the cinacalcetbased regimen, where effectiveness is the proportion of subjects reaching an iPTH level of 150-300 pg/mL."

Should read:

"Figure 1 is a scatterplot of all 1,000 bootstrap replicates, with the $x$-axis representing the incremental effectiveness and the $y$-axis representing the incremental cost of the paricalcitol-based regimen compared with the cinacalcetbased regimen, where effectiveness is the proportion of subjects reaching an iPTH level of $150-300 \mathrm{pg} / \mathrm{mL}$." 